\section{División sexual del trabajo en Uruguay en 2007 y 2013. Tendencias en los cambios y en las permanencias de las desigualdades de género.}

Sexual division of labor in Uruguay in 2007 and 2013. Trends in changes and permanence of gender inequalities

\section{KARINA BATTHYÁNY*}

SOL SCAVINO **

\section{Resumen}

El presente artículo busca dar cuenta de las tendencias de cambios o permanencias en las desigualdades de género en Uruguay entre 2007

Profesora Titular del Departamento de Sociología de la Facultad de Ciencias Sociales de la Universidad de la República. karina.batthyany@cienciassociales.edu.uy Dirección postal: Constituyente 1502 - Montevideo - 11200.

** Es Profesora Ayudante del Departamento de Sociología de la Facultad de Ciencias Sociales de la Universidad de la República.sol.scavino@cienciassociales.edu.uy Dirección postal: Constituyente 1502 - Montevideo - 11200. y 2013 a partir del análisis del tiempo dedicado por varones y mujeres al trabajo remunerado, al cuidado, las tareas domésticas y de voluntariado. En el país se han desarrollado una serie de políticas públicas para la modificación de las relaciones de género que aún no han impactado de manera clara en la división sexual del trabajo. Sin embargo, aparecen algunos aspectos del trabajo no remunerado que pueden ser ámbitos proclives para la modificación de la misma. El artículo busca dar respuesta a las siguientes preguntas: ¿Hay tendencias de cambio o permanencia en la división sexual del trabajo en Uruguay? ¿Cuáles son los tipos de trabajo no remunerado en los que se visualizan cambios? ¿Cómo se vinculan las desigualdades de género con la actual organización social del cuidado y las relaciones de género en el ámbito laboral?

Palabras clave: uso del tiempo, género, trabajo no remunerado, división sexual del trabajo.

\begin{abstract}
This article seeks to show the trends of change or permanence, of gender inequalities in Uruguay between 2007 and 2013 based on the analysis of time spent by men and women in paid work, care, housework and volunteer work. This country has developed a series of public policies and incentives for changing gender relations that have not yet clearly impacted on the sexual division of labor. However, some aspects of unpaid work seem to be prone areas where modification emerges. The article seeks to answer the following questions: Are there trends change or stay in the sexual division of labor in Uruguay? What are the types of unpaid
\end{abstract}


work in which changes are displayed? How gender inequalities are linked to the current social organization of care and gender relations in the workplace?

Key words: time use, gender, unpaid work, sexual division of labor.

\section{Introducción}

Uruguay, ha transitado un proceso de reconocimiento y visibilización de las desigualdades de género que se correspondió con una legitimación de las mismas como problema social. Este proceso ha sido vivido en gran parte de occidente y dentro del mismo en todos los países latinoamericanos, con distintas expresiones en cuanto a los avances en política, legislación e incorporación del tema como espacio de debate en el ámbito público.

En el caso uruguayo, en los últimos 10 años, se han llevado a cabo acciones, políticas y programas que buscan una transformación social que modifique las desigualdades de género presentes en la sociedad.

Dentro de estas acciones, se podrían mencionar importantes avances en los mecanismos formales que buscan instituir y legitimar la equidad de género en distintos ámbitos de la vida social (desde la creación del Instituto Nacional de las Mujeres, una nueva agenda de derechos que incluyó leyes que apuntan directamente a la modificación de algunos aspectos de las dinámicas de género, hasta la creación de un Sistema Nacional Integrado de Cuidados ${ }^{1}$ ).

En 2005 se ha creado el Instituto Nacional de las Mujeres, que se encuentra en la órbita del Ministerio de Desarrollo Social cuyo nombre y cometidos fueron definidos en la Ley $N^{\circ} 17930$ artículo
Debido al vínculo que existió desde un principio entre la academia, la sociedad civil organizada y el Estado, los cuidados han sido vistos como un problema social, demográfico, económico en el que las desigualdades de género son centrales (Aguirre, et al, 2014). Tanto es así, que en sus objetivos principales, dicho sistema se plantea la modificación de la actual división sexual del trabajo (Art. 9 de la referenciada Ley).

Este conjunto de elementos muestra algunos de los avances en Uruguay, país en el que la equidad de género se ha tornado un tema político y público.

Al mismo tiempo, se han creado conceptos y metodologías que permiten dar cuenta de distintas dimensiones de las desigualdades de género. La proliferación de las Encuestas de Uso del Tiempo en la región han permitido dar cuenta de cómo es la división sexual del trabajo en las distintas sociedades.

En el caso Uruguayo se cuenta actualmente con dos mediciones (2007 y 2013) que se busca observar en el presente trabajo para poder conocer tendencias en cambios o permanencias en la división sexual del trabajo entre varones y mujeres.

377 que está encargado de regular y monitorear las políticas de género en Uruguay, así como transversalizar dicha perspectiva. Además se han aprobado importantes leyes como la Ley 18.476 que estableció para las elecciones de 2014 una cuota de género como mecanismo de acceso de las mujeres en el poder político, exigiendo una mayoría especial de dos tercios; Ley 18.987 de "Interrupción voluntaria del embarazo", Ley n 19. 167 que tiene por objetivo "regular las técnicas de reproducción humana asistida acreditadas científicamente, así como los requisitos que deben cumplir las instituciones públicas y privadas que las realicen", Ley 19.075 de "Matrimonio Igualitario" se creó la posibilidad de contraer matrimonio entre parejas del mismo sexo en Uruguay; Ley 19 353, de Cuidados, estableció a los cuidados como un derecho de la población y como un deber a asumir de manera corresponsable entre los distintos sectores (Estado, Mercado, Comunidad y Familias) y entre varones y mujeres. 
De esta manera, se podría considerar si los cambios a nivel discursivo y de acciones políticas están siendo acompañados por cambio en los roles de género, conocidos en este caso a través de las tareas y trabajos que realizan varones y mujeres en Uruguay, así como del tiempo que dedican a cada uno de los mismos y los costos o beneficios que los mismos ofrecen en términos de autonomía y pleno ejercicio de derechos sociales, ciudadanos.

\section{Rupturas epistemológicas: trabajo a la luz de la perspectiva de género.}

Los estudios de género, al ubicarse como teorías críticas dentro de las ciencias sociales, han requerido una serie de rupturas epistemológicas y redefiniciones necesarias para poder construir su objeto de estudio.

En primer lugar, se destaca la ruptura con el concepto tradicional de trabajo como sinónimo de empleo. El concepto de trabajo se amplió a raíz de las reflexiones realizadas por académicas feministas sobre el carácter y el valor de las tareas domésticas y de cuidados, que permitieron visualizarlas como parte del primero. Dentro de los variados aportes, en la década de 1980 se realizan una serie de estudios sobre el trabajo de cuidados (Balbo (1980), Bimbi (1985) y Saraceno (1980) en Carrasco, Boderías y Torns, 2011: 32-36).

Las críticas al concepto de trabajo y su visibilidad, según Himmelweit (1995) por su homologación al concepto de trabajo fabril fueron tan impactantes que años más tarde, autores varones de renombre como Giddens, incorporaron estos elementos en los diccionarios básicos de sociología. Dicho autor señala que el trabajo "puede definirse como la ejecución de tareas que suponen un gasto de esfuerzo mental y físico, y que tienen como objetivo la producción de bienes y servicios para atender a las necesidades humanas" (Giddens, 2000: 397). Estas revisiones conceptuales no solo se desarrollaron en el ámbito académico, sino que pronto, organismos reguladores del trabajo la incorporaron. Por ejemplo, la Organización Internacional del Trabajo (OIT), también ha adoptado una noción amplia del concepto de trabajo que lo define como "todas las actividades realizadas en unidades económicas por personas de cualquier sexo con el fin de producir bienes o servicios para el consumo de terceros o el autoconsumo" (OIT, 2013:27).

En segundo lugar, la ampliación del concepto, se vio acompañada de cambios en los estudios sobre los regímenes de bienestar social. Tradicionalmente, este tipo de estudios se centraban básicamente en el aporte del Estado y el mercado como generadores de bienes y servicios. Sin embargo, esta estructura binaria se fue modificando por la integración de otros sectores en el análisis de los regímenes de bienestar social. Por ejemplo, se incorporaron a las familias como tercer sector relevante en la producción de bienes y servicios para el bienestar social ${ }^{2}$ (Esping-Andersen, 2000) y también a la comunidad (Adelantado et al., 1998:130-133 que toma los aportes de Hill (1996) y Pijl y Ungerson (1994)). Desde entonces el Estado, mercado, hogares y comunidad

En sus primeros estudios, Esping-Andersen no consideraba a la familia como uno de los sectores proveedores de bienestar y no integraba en su esquema las dificultades de la forma de provisión de bienes y servicios que provenían de un modelo en el que las mujeres veían comprometido el ejercicios de su ciudadanía social, por la dependencia de un marido trabajador (o similar) que viabilizaba sus derechos sociales a través del trabajo remunerado (Pateman, 1990; Saraceno, 1995). Sin embargo, luego de las críticas recibidas por académicas feministas, por ejemplo Lewis (1997), modificó su modelo integrando la esfera familiar. 
se presentan como los cuatro sectores más relevantes en la producción de bienes y servicios para el bienestar social y el tipo de intercambio que se da entre ellos y su carácter mercantil o gratuito determinan el tipo de bienestar del sistema social que se esté estudiando.

Varios de los análisis de los regímenes de bienestar se centran en el vínculo de las personas con el mercado laboral y el acceso de derechos a través de él; en la asignación colectiva de recursos y en la división sexual del trabajo (Esping-Andersen, 2000), existe un consenso en que los mismos se apoyan en y se construyen bajo el supuesto de una familia tradicional en la que los varones son los proveedores económicos y las mujeres son cuidadoras y realizadoras del trabajo domésticos (EspingAndersen, 1990; Lewis, 1997; Sainsbury, 2000).

Aguirre $(2003)^{3}$ clasifica a los estados como familistas (la responsabilidad del bienestar es de las familias y las mujeres) y desfamiliarizadores (hay una derivación de funciones de cuidado, tareas domésticas hacia el estado y mercado). Este último modelo permite pensar la corresponsabilidad entre Estado, mercado, comunidad y hogares, en la realización de las tareas necesarias para proveer de bienes y servicios a la población pero particularmente para generar una nueva organización social del cuidado.

El modelo familista, el cual se asocia a una división sexual del trabajo en donde las mujeres son las encargadas de los cuidados y de las tareas domésticas ${ }^{4}$ (además de proveer ingresos

La autora sigue a Sarraceno y Sainsbury en esta línea de análisis de Estados de Bienestar. por el trabajo remunerado) y los varones se dedican fundamentalmente a la provisión económica mediante el trabajo remunerado es un aspecto presente en la mayor parte de los países Latinoamericanos.

Según Martínez y Voorend (2013) son los preponderantes en Latinoamérica. Lejos de estimular la corresponsabilidad entre sectores, los Estados en la región cuestionan poco el rol de las mujeres en el trabajo no remunerado de cuidados o directamente se basan en él para la aplicación de las políticas públicas y la generación de productos necesarios para el bienestar de la población.

En tercer lugar, en el ámbito económico, acompañando la ruptura conceptual y redefinición del trabajo, también se redefinieron los conceptos de producción y de frontera general de la producción. La producción pasó a ser considerada una actividad en la que un agente económico suministra bienes y servicios a otras unidades mediante la utilización de insumos. Esto significa que al igual que en la economía de mercado, los servicios no remunerados para los propios miembros del hogar (domésticos y de cuidado de personas), servicios no remunerados para otros hogares y para la comunidad y servicios voluntarios ${ }^{5}$ no remunerados forman parte de la Frontera General de Producción (Gómez Luna, 2010:24-26).

manera no remunerada. Este tipo de trabajo se nominó de diversas formas: haciendo énfasis en su vínculo con la reproducción de la sociedad y de la vida se lo ha llamado "trabajo reproductivo", haciendo énfasis en su carácter gratuito, desvinculado de los derechos canalizados a través del mercado laboral, se lo ha denominado "Trabajo no remunerado"

5 El trabajo voluntario refiere a aquel que produce un bien o servicio para la comunidad, pero que no es remunerado, piénsese en trabajo voluntario en merenderos infantiles, hogares de ancianos, comúnmente realizados a través de organizaciones de la sociedad civil o religiosas. 
En el ámbito de las ciencias sociales, junto a la revisión del concepto de trabajo, surgió la necesidad de crear nuevos instrumentos metodológicos para dar cuenta empíricamente de la magnitud del mismo, las características de las personas que lo realizan y las relaciones sociales existentes en torno al mismo, por ejemplo, la evidencia de las desigualdades de género en su realización. Para llevar a cabo dicha empresa, la medición del tiempo dedicado al trabajo doméstico, de cuidados y voluntario no remunerado fue una de las metodologías más desarrolladas y consensuadas por académicos y técnicos de la región, inspirados mayoritariamente en los antecedentes europeos y norteamericanos (Araya, 2003).

Las Encuestas de Uso del Tiempo son herramientas diseñadas para captar al trabajo no remunerado. El mismo, suele ser definido por ser un trabajo por el cual no se recibe ningún tipo de remuneración, o ganancia en especias, favores o trueques. Se ha identificado que es mayoritariamente realizado por mujeres y en el marco de los hogares (Aguirre, 2009; Batthyány, 2015). Contempla una gran variedad de actividades como: tareas domésticas, tareas de cuidados, trabajo voluntario y trabajo de ayuda para otros hogares ${ }^{6}$.

La medición del uso del tiempo, permite visibilizar si existen diferencias en el comportamiento de varones y mujeres, sus características y relación con otros fenómenos socialmente relevantes como las desigualdades de clase, las desigualdades en el mercado laboral, el sistema

\footnotetext{
Para ver una descripción detallada de cada una de sus definiciones y cada uno de los ítems considerados para su medición, se sugiere consultar Batthyány, K (Coord). 2015. Los tiempos del bienestar social. Género, trabajo no remunerado y cuidados en Uruguay. Doble Clic Editoras, Montevideo.
}

educativo, el tipo de zona donde residen, la convivencia con personas dependientes, entre otros. A través de este instrumento se han visibilizado importantes desigualdades sociales de género, que han puesto de manifiesto las limitaciones que tienen las mujeres en el goce de una plena ciudadanía social y derechos. También, los estudios de uso del tiempo permiten la realización de ejercicios de valorización económica del trabajo no remunerado (Gómez Luna, 2003; Durán, 2006; OPS-CEPAL-CSIC, 2008; Salvador, 2009 y 2015) que son insumos para cálculos de cuentas satélites vinculadas a las cuentas nacionales y cálculos alternativos del Producto Bruto Interno. Su medición ha derivado también en ejercicios de valorización económica del tiempo de trabajo remunerado y no remunerado.

Si bien la medición del tiempo como un tiempo lineal y objetivo recibe varias críticas (Belloni (1988), Durán (2002), Grossin (1998), Gershuny y Sullivan (1998) en Delfino, (2009); Legarreta, M.I., 2011) y se pueden pensar otras a partir de Elías $^{7}$ (1989); su expandida aplicación se explica en parte por la virtud de captar un tiempo comparable entre personas y países que permite la captación de desigualdades estructurales de género.

\footnotetext{
A diferencia de Kant, quien considera que tiempo y espacio son categorías a priori en el conocimiento, Elías plantea que el tiempo es una construcción social que muestra claramente cómo funciona la introyección de los acuerdos y significaciones construidas intersubjetivamente y en contextos determinados. En palabras del autor "la transformación de la coacción externa de la institución social del tiempo en una pauta de autocoacción que abarca toda la existencia del individuo es un ejemplo gráfico de la manera en que un proceso civilizador contribuye a modelar una actitud social que forma parte integrante de la estructura de la personalidad del individuo" (Elías. N. 1989:21)
} 


\section{Objetivos y Metodología.}

El presente trabajo tiene por objetivo general comparar las tendencias en la distribución y dedicación del tiempo de varones y mujeres al trabajo remunerado y no remunerado en Uruguay en dos momentos del tiempo (2007 y 2013). Dentro de los objetivos específicos se encuentran conocer cómo se distribuye y cuánto tiempo se dedica a cada uno de los componentes del trabajo no remunerando en 2007 y 2013.

La metodología es de tipo cuantitativa y consiste en la comparación de los resultados de las Encuestas de Uso del Tiempo 2007 y 2013 en Uruguay, en términos de tendencias generales, puesto que las encuestas son comparables solo para grandes grupos poblacionales y no así para grupos desagregados por pequeños cambios en la muestra. El Instituto Nacional de Estadística de Uruguay se ha encargado de ponderar las bases luego de realizada la encuesta para garantizar las condiciones de comparabilidad de los datos en grandes poblaciones. Sin embargo, se ha optado por interpretar en términos de tendencias en el presente trabajo. Sin duda, garantizar la comparabilidad de las Encuestas de Uso del Tiempo a nivel nacional y regional es un desafío que aún persiste y que requiere de compromisos políticos, económicos y técnicos sostenidos en el tiempo. Sumado a esto, tal y como se mencionará al final del presente artículo, cada vez es más imperiosa la necesidad de contar con datos panel de uso del tiempo para enriquecer el análisis y complejizar reflexiones. Las características de ambas encuestas han sido descritas en la parte IV.

Dentro de las limitaciones del estudio, se destaca como la más importante la dificultad en la comparación de ambas encuestas por cambios introducidos en el instrumento aplicado en 2013, en relación a 2007. Entre estos cambios podrían mencionarse: el aumento del número de preguntas e ítems medidos de cuidados; el desfasaje entre la aplicación de la Encuesta de Uso del Tiempo 2013 y la aplicación de la Encuesta Continua de Hogares 2013 (en base a la cual se obtienen todos los datos de hogares y personas no referidos particularmente al uso del tiempo y el trabajo no remunerado) y cambios en la metodología para calcular a partir del tiempo diario el tiempo semanal en 2007 y 2013.

Si bien el Instituto Nacional de Estadística ha generado ponderadores correctores de la base ex post facto en busca de la comparación, se ha elegido en este trabajo considerar solamente los números que refieren a grandes poblaciones ${ }^{8}$, como tendencias generales para intentar disminuir al máximo la posibilidad de que los cambios observados en el fenómeno puedan asociarse a los instrumentos de medida.

Se actúa bajo el supuesto de que a menor tamaño de la muestra, aumenta el error que puede provenir por la diferencia en los instrumentos. 
Recuadro 1. Características generales de las Encuestas de Uso del Tiempo en Uruguay en 2007 y 2013.

\begin{tabular}{|c|c|c|}
\hline & 2007 & 2013 \\
\hline $\begin{array}{l}\frac{7}{0} \\
\frac{\mathbb{O}}{\bar{O}} \\
\frac{\mathbb{T}}{0} \\
\frac{0}{2}\end{array}$ & $\begin{array}{c}\text { Módulo de la } \\
\text { Encuesta Continua } \\
\text { de Hogares }(\mathrm{ECH})\end{array}$ & $\begin{array}{l}\text { Módulo de la } \\
\text { Encuesta Continua } \\
\text { de Hogares }(\mathrm{ECH})\end{array}$ \\
\hline 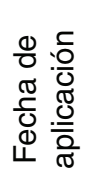 & $\begin{array}{c}\text { Setiembre de } 2007 \text {, } \\
\text { junto a la ECH de } \\
\text { ese mismo mes. }\end{array}$ & $\begin{array}{c}\text { Entre mayo y } \\
\text { agosto de } 2013 \text { a } \\
\text { los hogares que se } \\
\text { había realizado la } \\
\text { ECH en marzo }\end{array}$ \\
\hline 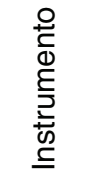 & $\begin{array}{l}\text { Cuestionario-Lista } \\
\text { de actividades } \\
\text { precategorizada }\end{array}$ & $\begin{array}{c}\text { Cuestionario-Lista } \\
\text { de actividades } \\
\text { precategorizada }\end{array}$ \\
\hline 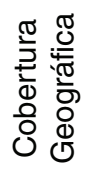 & $\begin{array}{c}\text { Montevideo y } \\
\text { localidades con más } \\
\text { de } 5000 \text { habitantes }\end{array}$ & $\begin{array}{c}\text { Montevideo y } \\
\text { localidades con más } \\
\text { de } 5000 \text { habitantes }\end{array}$ \\
\hline 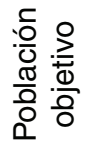 & $\begin{array}{l}\text { Personas de } 14 \text { o } \\
\text { más años de edad }\end{array}$ & $\begin{array}{l}\text { Personas de } 14 \text { o } \\
\text { más años de edad }\end{array}$ \\
\hline 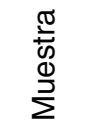 & 4200 hogares & $\begin{array}{c}3.356 \text { hogares } 7.447 \\
\text { personas }\end{array}$ \\
\hline 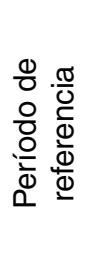 & $\begin{array}{c}\text { Último día } \\
\text { laborable y último } \\
\text { día no laborable } \\
\text { identificándose la } \\
\text { cantidad de minutos } \\
\text { y horas para cada } \\
\text { uno de los casos. }\end{array}$ & $\begin{array}{c}\text { Día anterior } \\
\text { (laborable o no } \\
\text { laborable). }\end{array}$ \\
\hline
\end{tabular}

Fuente: elaborado en base a metadatos de las Encuestas de Uso del Tiempo 2007 y 2013 del Instituto Nacional de Estadística.
Como es posible apreciar en el Recuadro 1, a medida que se fueron realizando distintas mediciones en el país, las mismas fueron cambiando de modalidad en sus fechas de aplicación, su cobertura geográfica (ganando en extensión de cobertura a mayor cantidad de centros urbanos del país) y ampliando también la muestra para el logro del objetivo anterior.

A continuación, presentaremos algunos de los indicadores básicos de uso del tiempo en varones y mujeres uruguayas centrándonos en el análisis de las diferentes tendencias que muestran los datos en los cortes transversales 2007-2013.

\section{Principales datos sobre el Uso del tiempo y división sexual del trabajo en Uruguay 2007 y 2013}

En función del concepto amplio de trabajo definido, se analizará el uso del tiempo de varones y mujeres en el trabajo remunerado y no remunerado, tomados conjuntamente y por separado para dar cuenta de cómo se distribuye el mismo entre la población uruguaya tanto en 2007 como en 2013.

\subsection{Carga Global de Trabajo 2007-2013}

La Carga Global de Trabajo es uno de los indicadores que permite medir la magnitud del trabajo total, incluyendo al trabajo remunerado y no remunerado. Ofrece una mirada completa sobre el trabajo y la división sexual del trabajo que vista solo a través del trabajo remunerado o solo a través del no remunerado, podría conducir a sesgos en el análisis. Por ejemplo, si se observara solo el trabajo remunerado, se podía concluir de manera errada que son los varones los que 
participan y dedican más tiempo al trabajo. Las tasas de actividad en el trabajo remunerado son mayores en los varones que en las mujeres $(74,3$ $\%$ y $55,9 \%$ con una brecha de $18,4 \%$ según INE, 2015:105). Sin embargo, esto no es así cuando se trata del trabajo no remunerado, sino que sucede la situación inversa.

Como ya se ha planteado, el trabajo también integra otras actividades como el trabajo de cuidados, doméstico y voluntario que se da en los hogares y entre los mismos. Cuando todas las horas de trabajo son tomadas en cuenta, aparece que son las mujeres las que realizan los mayores aportes a la producción de bienes y servicios para el bienestar social, como muestra la Tabla 1 a continuación.

Tabla 1. Distribución porcentual de la carga global de trabajo por sexo. Total país, 2007 y 2013.

\begin{tabular}{|l|l|l|}
\hline & 2007 & 2013 \\
\hline Mujeres & 52,3 & 53,1 \\
\hline Varones & 47,7 & 46,9 \\
\hline
\end{tabular}

Fuente: elaboración propia en base a los microdatos de la Encuesta de Uso del Tiempo y Trabajo No Remunerado 2007 y 2013, módulos de la Encuesta Continua de Hogares, INE-FCSINMUJERES. Uruguay.

Este primer indicador, muestra que tanto para 2007 como para 2013 las mujeres aportan más del $50 \%$ de la carga global de trabajo en Uruguay y que en 2013 los porcentajes se mantienen casi incambiados respecto a 2007. Aumenta levemente la carga en las mujeres en tan solo un $1 \%$. Es decir que en el período de 6 años entre 2007 y 2013 no se registran cambios significativos en la Carga Global de Trabajo por sexo en Uruguay.
En la distribución del peso del tiempo dedicado a cada uno de los dos componentes de trabajo (TR y TNR) no ha variado significativamente entre 2007 y 2013, como puede observarse en la Tabla 2 a continuación.

Tabla 2. Distribución porcentual de la carga global de trabajo de la población de 14 años de edad y más. Total país, 2007 y $2013^{9}$.

\begin{tabular}{|c|c|c|}
\hline & 2007 & 2013 \\
\hline TNR & 49 & 49,9 \\
\hline TR & 51 & 50,1 \\
\hline
\end{tabular}

Fuente: elaboración propia en base a los microdatos de la Encuesta de Uso del Tiempo y Trabajo No Remunerado 2007 y 2013, módulos de la Encuesta Continua de Hogares, INE-FCSINMUJERES. Uruguay.

\subsection{División sexual del trabajo en 2007 y 2013}

Las cargas de trabajo remunerado y no remunerado por sexo, son un indicador de la división sexual del trabajo, es decir, de cómo se reparten las tareas del ámbito productivo y reproductivo entre varones y mujeres.

Esto es importante debido a que las dedicaciones diferenciales de tiempo a unas tareas y otras hablan del tipo de tareas que están socialmente asignadas a unos y otros. Partiendo de la base de que el gusto es algo construido socialmente y no es algo innato, se recuerda que la socialización de género produce

La carga global de trabajo es un cálculo porcentual en base a la cantidad de tiempo (no a la participación) que la población dedica al trabajo remunerado y no remunerado (cuando es por sexo, el total de horas se pondera por esta misma variable, de forma tal de que el peso de varones y mujeres en la población no influya en la cantidad de horas dedicadas a las tareas). 
ámbitos de actuación y gustos asociados a los mismos distintos entre varones y mujeres. Los mismos tienen status sociales jerárquicos y el trabajo doméstico y de cuidados son carentes del valor social (Torns, 2008) que porta el trabajo remunerado (aunque es claro que hay una gran heterogeneidad en los valores del trabajo remunerado según de cuál se trate).

Mediante la realización de trabajo no remunerado, no se accede a remuneraciones ni derechos sociales. Gran parte de las tareas de trabajo no remunerado son rutinarias y cotidianas, esenciales para reproducir la vida cotidiana e implican compromisos morales y éticos subjetivados particularmente en las mujeres, que hacen que sea un tipo de trabajo irrenunciable para varias de ellas.

En términos generales, cuando observamos a qué tipo de trabajo se dedican varones y mujeres, vemos que los primeros lo hacen mayoritariamente al trabajo remunerado y las segundas al no remunerado, obedeciendo a los patrones de la clásica división sexual del trabajo en occidente en la que los varones son los encargados de la provisión económica y las mujeres cuidadoras y encargadas del ámbito doméstico (Beck y BeckGernsheim, 2012; Arriagada, 1997).

En Uruguay, la organización del trabajo entre varones y mujeres se presenta siguiendo este tipo de división sexual del trabajo (aunque, como se ha evidenciado para gran parte de la región, las mujeres se han incorporado activamente al mercado laboral, lo cual introdujo tensiones entre el ámbito doméstico y laboral que recaen específicamente sobre ellas ya que no existe una apropiación por parte de los varones del trabajo doméstico y de cuidados, pero si una incorporación de las mujeres al trabajo remunerado, lo que hace que tengan una doble carga laboral y menos tiempo disponible para actividades de ocio, descanso, entre otras ${ }^{10}$ ). Dicha división sexual del trabajo, se expresa en el porcentaje del tiempo que los varones y las mujeres dedican al trabajo remunerado (TR) y al trabajo no remunerado (TNR). Como muestra la Tabla3, a continuación, los varones efectivamente dedican una mayor proporción del tiempo al TR (72\% y 68\%) y las mujeres al TNR (65\%). A su vez, dicha distribución del tiempo en los varones y las mujeres se mantiene relativamente incambiada en el período 2007-2013.

Tabla 3. Distribución porcentual del tiempo dedicado al trabajo remunerado y no remunerado por sexo. Total país, 2007 y 2013

\begin{tabular}{|c|c|c|c|c|c|c|}
\hline & \multicolumn{3}{|c|}{ Varones } & \multicolumn{3}{c|}{ Mujeres } \\
\cline { 2 - 7 } & 2007 & 2013 & $07-13$ & 2007 & 2013 & $07-13$ \\
\hline TNR & 28 & 32 & 4 & 65 & 65 & 0 \\
\hline TR & 72 & 68 & -4 & 35 & 35 & 0 \\
\hline Total & 100 & 100 & & 100 & 100 & \\
\hline
\end{tabular}

Fuente: elaboración propia en base a los microdatos de la Encuesta de Uso del Tiempo y Trabajo No Remunerado 2007 y 2013, módulos de la Encuesta Continua de Hogares, INE-FCSINMUJERES. Uruguay.

Del trabajo total de las mujeres, en 2007 el $65 \%$ del tiempo estaba dedicado al trabajo no remunerado registrándose para 2013 el mismo porcentaje de tiempo utilizado para el TNR $(64,6 \%)$, mientras que los varones ocupaban

\footnotetext{
Es extensa la literatura que ha abordado el problema de la doble carga laboral de las mujeres, de lo que se han dado en llamar problemas de "conciliación" trabajo-familia. Dos ejemplos para ampliar la lectura son Faur (2014) y Tobío (2005),
} 
$28 \%$ de su tiempo al trabajo no remunerado en 2007 y $32(31,9 \%)$ en 2013.

Este es uno de los principales indicios de cómo es la división sexual del trabajo en Uruguay, en la cual se evidencia que las mujeres realizan mayoritariamente tareas en el hogar y de cuidados mientras que los varones se vuelcan más al trabajo pago en el ámbito productivo. También, el análisis de los datos sugiere una tendencia a la permanencia en el tiempo.

Los datos muestran que los varones aumentaron cerca de un $4 \%$ la magnitud de tiempo de TNR mientras que las mujeres mantuvieron una proporción similar de tiempo dedicado al trabajo remunerado y no remunerado en ambos períodos.

Por otra parte, en cuando al porcentaje de tiempo dedicado al trabajo remunerado por mujeres y varones, se observa una leve redistribución entre 2007 y 2013 entre varones y mujeres del mismo (las mujeres dedican 2\% más de su tiempo al trabajo remunerado en 2013 que en 2007).

Gráfico 1. Distribución porcentual dedicada al trabajo remunerado y al trabajo no remunerado por varones y mujeres. Total país, 2007 y 2013.

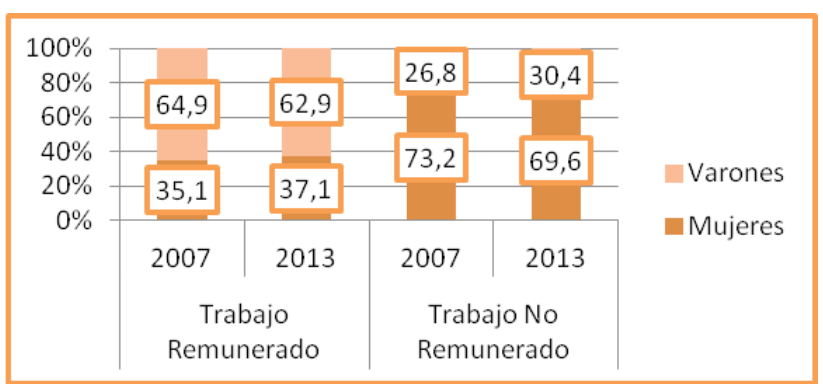

Fuente: elaboración propia en base a los microdatos de la Encuesta de Uso del Tiempo y Trabajo No Remunerado 2007 y 2013, módulos de la Encuesta Continua de Hogares, INE-FCSINMUJERES. Uruguay.
Si bien se trata de una porcentualización en base al tiempo que aportan las mujeres y los varones de trabajo remunerado y no de su participación, al ser una modificación para las mujeres en sentido positivo, este indicador podría vincularse con el aumento de la población económicamente activa femenina registrados en Uruguay (Inmujeres, 2013) lo que significaría un incremento en la participación de trabajo remunerado más importante en las mujeres que en los varones.

Como ya se ha dicho, los varones dedican 3,6\% más de tiempo al trabajo no remunerado que en 2007. Si esta observación se mantuviera en futuras mediciones, se podría comenzar a hablar de una leve y paulatina tendencia al aumento de la dedicación de tiempo de TNR en la población masculina. Aunque por ahora, los datos hablan más bien de una reproducción del escenario captado en 2007 , que de grandes modificaciones respecto al mismo.

Sin embargo, los cambios en las tasas de empleo entre 2006 y 2014 por sexo muestran que si bien el empleo de las mujeres ha aumentado más que el de los varones en el período considerado, persiste una desigualdad de género en la participación en el mercado de empleo, evidenciada por el valor de la brecha de género en la tasa de empelo (19,3\% más de varones $)^{11}$.

En sentido opuesto, la participación de varones y mujeres en el trabajo no remunerado ha disminuido en el período $(7,7 \%)$ y lo ha hecho de mayor manera en los varones $(-9,7 \%)$ que en las mujeres $(-5,6 \%)$. Es decir que mientras que la participación en el mercado de empleo ha aumentado en el período, potenciado por

\footnotetext{
Instituto Nacional de Estadística de Uruguay sobre Tasa de actividad, empleo y desempleo, revisados en el año 2013.
} 
el crecimiento económico en el país ${ }^{12}$, entre otros factores, la participación en el trabajo no remunerado ha disminuido. Esto abre preguntas acerca de cómo son los cambios en la participación en los tipos de trabajo (TR y TNR) y si los mismos obedecen a cambios coyunturales (económicos y sociales) o si se están modificando los parámetros culturales en torno a la realización del trabajo no remunerado. Esto a su vez implica examinar cada uno de los componentes del trabajo no remunerado para conocer en cuáles de ellos merma la participación y cuáles no.

Tabla 4. Tasa de empleo en el mercado de empleo y tasa de participación en el trabajo no remunerado* por sexo en 2007 y 2013. Brechas de género y en el período. Total país.

\begin{tabular}{|c|c|c|c|c|}
\hline \multicolumn{5}{|c|}{ Trabajo Remunerado (tasa de empleo) } \\
\hline & Mujeres & Varones & Total & $\begin{array}{c}\text { Brecha } \\
\text { V/M }\end{array}$ \\
\hline 2007 & 46,1 & 69,1 & 56,7 & 22,9 \\
\hline 2013 & 50 & 70,2 & 59,5 & 20,2 \\
\hline $\begin{array}{c}\text { Brecha } \\
13-07\end{array}$ & 3,8 & 1,1 & 2,8 & $-2,8$ \\
\hline Trabajo No Remunerado (tasa de participación) \\
\hline \multicolumn{5}{|c|}{} \\
\hline $\begin{array}{c}\text { Mujeres } \\
2007\end{array}$ & 95,7 & 85,4 & 91 & $-10,3$ \\
\hline 2013 & 90,1 & 75,7 & 83,3 & $-14,4$ \\
\hline $\begin{array}{c}\text { Brecha } \\
13-07\end{array}$ & $-5,6$ & $-9,7$ & $-7,7$ & 4,1 \\
\hline
\end{tabular}

Fuente: elaborado en base a los datos del Instituto Nacional de Estadística de Uruguay sobre Tasa de actividad, empleo y desempleo, revisados en el año 2013 y elaboración propia en

\footnotetext{
Pueden consultarse las cifras en torno al crecimiento económico del Uruguay en el Instituto Nacional de Estadística (www.ine.gub. uy) y en las cifras del Banco Mundial para Uruguay (http://www. bancomundial.org/es/country/uruguay/overview)
}

base a los microdatos de la Encuesta de Uso del Tiempo y Trabajo No Remunerado 2007 y 2013, módulos de la Encuesta Continua de Hogares, INE-FCS-INMUJERES. Uruguay. * Las diferencias de proporciones entre varones en los dos años son estadísticamente significativas $(X 2=104,489$, gl 1 , Sig. , 000) y también entre mujeres en ambos años (X2 =95,468, gl 1, Sig. ,000)

Así se ve como la tasa de participación se comporta diferente al tiempo promedio dedicado al trabajo no remunerado y remunerado. En la Carga Global de Trabajo, las magnitudes de porcentaje de tiempo dedicado al trabajo remunerado y no remunerado son similares en el período, con una levísima tendencia al aumento del tiempo dedicado al trabajo no remunerado. Sin embargo, en cuanto a la participación en el trabajo remunerado y no remunerado, se registra una importante disminución en la participación de la población uruguaya en el TNR $(7,7 \%)$ y un aumento de la participación en el trabajo remunerado $(2,8 \%)$.

La disminución de la participación del trabajo no remunerado, podría estar relacionada con la mercantilización de las tareas domésticas y de cuidados que permite que las personas externalicen este tipo de trabajo, comprándolo en el mercado como un servicio. Sin embargo, la misma no asegura una división sexual del trabajo justa, ni un reparto corresponsable de las tareas domésticas y de cuidados restantes al interior del hogar.

Tanto varones como mujeres han disminuido su participación en el TNR en el período. Pero han sido los varones los que más lo han hecho, reduciendo su participación casi 10\% en relación a 2007 mientras que las mujeres lo hicieron en casi $6 \%$.

Esto implica que en vez de incorporarse progresivamente al TNR, los varones participan 
menos del mismo en 2013 que en 2007. Sin embargo, como veremos a continuación, entre los varones que se involucran activamente en el trabajo no remunerado, se registra un incremento en el tiempo promedio semanal dedicado al mismo.

En relación al trabajo no remunerado, es posible establecer que si bien la participación en el mismo ha disminuido en el período $(7,7 \%)$ el tiempo semanal promedio dedicado al mismo ha aumentado en 3 horas, pasando de dedicarse 27 en total a 30 horas semanales. Cuando observamos quiénes han aumentado de manera más pronunciada el tiempo semanal dedicado al trabajo no remunerado en el período, vemos que son los varones (aumentaron 4 horas promedio semanales en relación a la medición de 2007). Las mujeres también han aumentado 1 hora semanal promedio de TNR respecto a 2007 , lo que representa una reducción en 3 horas de la brecha de género de tiempo dedicado al TNR en el período.

El aumento del tiempo promedio otorgado por los varones, se registra cuando se integran solo las personas que participan activamente del trabajo no remunerado y cuando se toma en cuenta al total de la población mayor de 14 años (potencialmente realizadora de TNR).

Cuando se considera en la población uruguaya a las personas mayores de 14 años que participan y las que no participan del trabajo remunerado ${ }^{13}$ pasa lo mismo que se registra cuando se considera a la población que solo participa del TNR, la brecha de género en el período disminuye en 2,4 horas semanales.

Quienes no realizan TNR aportan 0 hora semanal al mismo y quienes si lo hacen, aportan más que 0 hora semanal al trabajo no remunerado.
Si bien es positivo que los varones dediquen más tiempo al TNR, debemos recordar que la brecha de tiempo dedicado entre varones y mujeres es altísima, ya que las mujeres dedican una cantidad de tiempo semanal promedio que duplica al de los varones y es cercana a 40 horas semanales, lo que serían jornadas laborales extensas si fuesen ofrecidas en el mercado laboral (8 horas diarias de trabajo 5 días a la semana).

Así, vemos que son las mujeres quienes aportan mayor cantidad de tiempo de trabajo no remunerado tanto en 2007 (36 horas semanales promedio) como en 2013 (37 horas, Tabla 5). Esto muestra una persistente división sexual del trabajo y una carga de trabajo cotidiano en las mujeres superior a la de los varones, que recuerda el concepto de doble jornada laboral y los desafíos persistentes en torno a que los varones se involucren más y dediquen más tiempo al trabajo reproductivo (doméstico, de cuidados, voluntario).

Recordemos que si bien la brecha de género en el tiempo dedicado al trabajo no remunerado disminuye 3 horas promedio en el período, la brecha de género en la tasa de participación ha aumentado 4,1\% entre 2007 y 2013 ya que ambos disminuyen su participación respecto a 2007 , pero los varones lo hacen de manera más acentuada.

En relación al tiempo promedio, en 2007 las mujeres dedicaban 20 horas semanales promedio más de trabajo no remunerado que los varones. En 2013, las mujeres dedican 17 horas promedio más que los varones a este tipo de tareas. Esta reducción en la brecha se explica por un aumento del tiempo que dedican al trabajo no remunerado aquellos varones que 
efectivamente participan en el mismo. Como se dijo anteriormente, mientras que las mujeres mantienen su alto promedio de horas semanales dedicadas a estas tareas casi incambiado en el período (1 hora), los varones aumentaron 4 horas promedio.

Tabla 5. Tasa de participación (TP) y tiempo semanal promedio (HS) dedicado al trabajo no remunerado por sexo. Total país, 2007 y 2013*

\begin{tabular}{|c|c|c|c|c|}
\hline \multirow{2}{*}{} & \multicolumn{2}{|c|}{ Mujeres } & \multicolumn{2}{c|}{ Varones } \\
\cline { 2 - 5 } & TP & HS & TP & HS \\
\hline 2007 & 95,7 & 36 & 85,4 & 16 \\
\hline 2013 & 90,1 & 37 & 75,7 & 20 \\
\hline Dif. 07-13 & $-5,6$ & 1 & $-9,7$ & 4 \\
\hline \multirow{2}{*}{2007} & 91 & 27 & 10,3 & 20 \\
\cline { 2 - 5 } & TP & HS & TP & HS \\
\hline 2013 & 83,3 & 30 & 14,4 & 17 \\
\hline Dif. 07-13 & $-7,7$ & 3 & 4,1 & -3 \\
\hline
\end{tabular}

Fuente: elaboración propia en base a los microdatos de la Encuesta de Uso del Tiempo y Trabajo No Remunerado 2007 y 2013, módulos de la Encuesta Continua de Hogares, INE-FCSINMUJERES. Uruguay.

* Las diferencias de proporciones entre varones en los dos años son estadísticamente significativas ( $X 2=104,489$, gl 1, Sig. , 000) y también entre mujeres en ambos años $(X 2=95,468$, gl 1, Sig. ,000)

Dicho aumento del tiempo promedio dedicado por semana al trabajo no remunerado en el período en el caso de los varones, podría responder a varios factores. ${ }^{14}$ Entre ellos,

Uno de ellos podría estar relacionado a la disminución de la tasa de participación en el período (se "pierden" casos que trabajaban pocas horas, y aquellos varones que siguen participando son uno podría ser la presencia de cambios en el comportamiento de los varones en torno a algunas de las tareas del trabajo no remunerado. Por ejemplo, se encuentra que entre los varones que participan del TNR, hay un incremento del tiempo semanal promedio entre 2007 y 2013 en las tareas de cuidado (7 horas), en las tareas realizadas para otros hogares (9 horas) y en el trabajo voluntario (7 horas). Sin embargo, en el trabajo doméstico, no se registran cambios en el tiempo semanal promedio dedicado por los varones entre 2007 y 2013, como se mostrará de manera más exhaustiva en la siguiente sección. Mientras que mujeres y varones aumentan el mismo promedio de horas de trabajo no remunerado voluntario y para otros hogares, los varones aumentan el tiempo que dedican al trabajo de cuidados 3 horas promedio más que las mujeres. El cambio en la tasa de participación en el trabajo de cuidados entre los varones es de singo positivo, aunque su magnitud no es sustantiva $(0,8 \%)$.

En relación al aumento de las horas dedicadas al cuidado no remunerado y el leve aumento en su participación por parte de los varones, se encuentran en la literaturaespañola, antecedentes que indican que los varones y mujeres convergen lentamente en el tiempo dedicado a las tareas del hogar y el cuidado. El Instituto Nacional de Estadísticas español compara las cifras de las encuestas de Empleo del Tiempo en 2003 y 2009/2010 y destaca que si bien son las mujeres las que realizan la mayor parte del trabajo no remunerado, existe una leve convergencia en

los que dedicaban más tiempo al TNR, lo cual puede generar la "inflación" de las horas semanales promedio). El 10\% de diferencia en la tasa de participación masculina, puede relacionarse con la creciente posibilidad de comprar servicios de tareas domésticas y de cuidados, lo cual ofrecería una solución a aquellos a quienes les disgusta la realización de tareas domésticas, de cuidado o voluntarias, o no pueden hacerlo. 
el tiempo, aunque se subraya la abrumadora persistencia de desigualdades de género en la realización de las mismas (INE, 2015b) ${ }^{15}$. Por otra parte los estudios sobre nuevas paternidades y disposición de los varones hacia el cuidado infantil ponen de manifiesto que el trabajo de cuidados podría ser la puerta de entrada al mundo doméstico y del trabajo no remunerado para los varones (particularmente algunos presentes en la literatura anglosajona Schober, 2011; Snarey 1993 en Agirre Miguélez, 2016: 11).

En el caso Uruguayo, es claramente visible que la participación en el trabajo doméstico de los varones ha disminuido y el tiempo promedio dedicado a las tareas de trabajo doméstico entre aquellos que participan se ha mantenido incambiada, mientras que en el trabajo de cuidados la participación es similar que en 2007, pero el tiempo promedio dedicado a los cuidados por parte de los varones ha aumentado significativamente.

Otro posible factor explicativo del cambio de las horas de TNR 2007 y 2013 puede no tener que ver con el cambio social o cultural en el fenómeno sino con el instrumento de medición. Entre la medición realizada en 2007 y la de 2013 la forma de preguntar sobre el trabajo de cuidados se especificó y amplió en la última medición con el fin de captar de manera más fina el fenómeno ${ }^{16}$. Esto puede generar un aumento

En España la tasa de participación de tareas domésticas y de cuidados de los hijos de los varones es $74.7 \%$ ( $5 \%$ más que la registrada en 2003), aunque el porcentaje sigue siendo menor al de ellas $(91.9 \%)$. La brecha de tiempo de trabajo no remunerado diaria cambió de 2 horas 54 minutos en 2003 a 2 horas, 13 minutos en 2010.

16 Los cuidados han sido medidos de manera más exhaustiva en 2013 que en 2007 y las muestras han sido tomadas de manera diferente, aunque corregidas con el fin de poder ser comparadas para grandes grupos. en las horas y participación declaradas puesto que las preguntas fueron más detalladas que en el formulario anterior.

Si bien mejoró la medición en 2013 sobre los cuidados en la población uruguaya, esta variante en el instrumento impide discernir de forma clara cuál es el origen del cambio.

\section{Variaciones en la dedicación a los diferentes componentes del trabajo no remunerado en Uruguay, 2007-2013.}

A continuación se analizaran los cambios y permanencias en el trabajo doméstico como componente del trabajo no remunerado.

\subsection{Trabajo doméstico no remunerado entre 2007 y 2013.}

Respecto a las brechas de género en el trabajo doméstico, es posible observar que entre 2007 y 2013 hay un aumento de 6,4\% de la brecha en la tasa de participación en las tareas domésticas, en detrimento de las mujeres. Es decir que son las mujeres las que se encargan de realizar el trabajo doméstico, indispensable para la supervivencia y sostenibilidad de la vida social. Se trata de un trabajo duro y estructurado si se considera la constancia que requiere y la dificultad de llevar a cabo la vida cotidiana sin su realización.

Sin embargo, es necesario destacar que por algunas circunstancias (como el ya mencionado aumento de su participación activa en el mercado laboral y la mayor mercantilización de los servicios domésticos) las mujeres han reducido su tiempo dedicado a las tareas domésticas entre 2007 y 2013, un promedio de 2 horas semanales. 
Tabla 6. Tasa de participación (TP) tiempo semanal promedio (HS) y brechas de género y en el período del trabajo doméstico no remunerado por sexo. Total país.

\begin{tabular}{|c|c|c|c|c|c|c|}
\hline \multirow{2}{*}{} & \multicolumn{2}{|c|}{ Mujeres } & \multicolumn{2}{c|}{ Varones } & \multicolumn{2}{c|}{$\begin{array}{c}\text { Diferencias } \\
\text { M/N }\end{array}$} \\
\cline { 2 - 7 } & TP & HS & TP & HS & TP & HS \\
\hline 2007 & 95,1 & 29 & 83,1 & 13 & 12 & 16 \\
\hline 2013 & 87,4 & 27 & 69,0 & 13 & 18,4 & 14 \\
\hline $\begin{array}{c}\text { Brecha } \\
13-07\end{array}$ & $-7,7$ & -2 & $-14,1$ & 0 & 6,4 & -2 \\
\hline
\end{tabular}

Fuente: elaboración propia en base a los microdatos de la Encuesta de Uso del Tiempo y Trabajo No Remunerado 2007 y 2013, módulos de la Encuesta Continua de Hogares, INE-FCSINMUJERES. Uruguay.*Las diferencias de proporciones entre varones en los dos años son estadísticamente significativas (X2 = 184,197, gl 1, Sig. , 000) y también entre mujeres en ambos años $(\mathrm{X} 2=153,319, \mathrm{gl}$ 1, Sig. ,000)

Este dato es muy importante, en la medida en que mostraría que la reducción de la brecha de género en el trabajo doméstico se debe a que las mujeres dedican menor cantidad de tiempo a las tareas domésticas (probablemente pagando a otra mujer para su realización) y no a que los varones hayan acentuado su participación. Esto mostraría, como ya se ha mencionado, que la mercantilización del trabajo doméstico no asegura una división sexual del trabajo más equitativa entre varones y mujeres ${ }^{17}$.

\footnotetext{
Si bien, como ya se ha mencionado, Uruguay es uno de los países en la región que más se ha preocupado en regularizar y dignificar el trabajo doméstico. En el mismo no se presentan mecanismos explícitos que contribuyan a modificar la actual división sexual del trabajo (por ejemplo, el trabajo doméstico está altamente feminizado, el 99,9\% son mujeres, como muestra la Tabla 8 en Anexos, frente a un $55 \%$ de mujeres en el total de la población ocupada). También, los salarios aún son pobres en relación a los de otros tipos de trabajo, 4 de cada 10 trabajadoras domésticas
}

En resumen, las tareas domésticas en Uruguay son realizadas mayoritariamente por mujeres, sean estas remuneradas o no, lo cual da una idea de los contratos de género vigentes, en los cuales las mujeres se encargan de los cuidados y el ámbito doméstico, mientras que los varones participan de otras tareas como el trabajo remunerado y dentro del mismo, trabajos más vinculados al dominio instrumental del mundo: los negocios, la ingeniería, producción agropecuaria, la milicia y altos cargos estatales, etc. (Inmujeres, 2015: 42).

\subsection{Trabajo de cuidados no remunerado entre 2007 y 2013.}

En relación al trabajo de cuidado entre 2007 y 2013 en Uruguay, se observa algo distinto a lo registrado en el caso del trabajo doméstico. La participación en el trabajo de cuidado, tanto en las mujeres como en los varones es mayor en 2013 que la observada en 2007 (2,6\% y 0,8\% según corresponda) al igual que el tiempo dedicado a estas tareas ( 4 y 7 horas semanales en promedio).

aún no aportan a la caja y casi 3 de cada 10 no cuentan con vacaciones, licencias por enfermedad o pago de licencias no gozadas. Sin embargo, se reconoce que el trabajo doméstico es una fuente de empleo y su regularización contribuiría a generar mayor visibilidad sobre el carácter de trabajo que tiene la realización de las tareas domésticas (sean estas pagas o no). Mediante intervenciones culturales, comunitarias y estatales, podría estimularse la participación de los varones en el trabajo doméstico remunerado (y no remunerado), lo que haría que, eventualmente la mercantilización del trabajo doméstico contribuya a la modificación de la división sexual del trabajo y los roles de género. La dignificación, formalización y valorización económica del trabajo doméstico podría contribuir a la valorización social (simbólica, cultural y también económica) del trabajo doméstico no remunerado, pero también de los otros tipos de trabajos no remunerados (por ejemplo, el de cuidados a dependientes). 
Tabla 7. Tasa de participación, tiempo promedio y brechas de género y en el período del trabajo de cuidados no remunerado por sexo. Total país.

\begin{tabular}{|c|c|c|c|c|c|c|}
\hline \multirow{2}{*}{} & \multicolumn{2}{|c|}{ Mujeres } & \multicolumn{2}{c|}{ Varones } & \multicolumn{2}{c|}{$\begin{array}{c}\text { Diferencias } \\
\text { M/V }\end{array}$} \\
\cline { 2 - 7 } & TP & HS & TP & HS & TP & HS \\
\hline 2007 & 32,5 & 18 & 23,5 & 10 & 9 & 8 \\
\hline 2013 & 35,1 & 22 & 24,3 & 17 & 10,8 & 5 \\
\hline $\begin{array}{c}\text { Brecha } \\
13-07\end{array}$ & 2,6 & 4 & 0,8 & 7 & 1,8 & -3 \\
\hline
\end{tabular}

Fuente: elaboración propia en base a los microdatos de la Encuesta de Uso del Tiempo y Trabajo No Remunerado 2007 y 2013, módulos de la Encuesta Continua de Hogares, INE-FCSINMUJERES. Uruguay. *las diferencias de proporciones para mujeres y varones en los dos años no son estadísticamente significativas.

Una posible forma de interpretar el aumento de las horas de trabajo de cuidado en países típicamente familistas (España, Italia, pero también Uruguay, Argentina, Chile), es la resistencia a la institucionalización del cuidado (infantil, de personas mayores o discapacitadas con dependencia), que también puede estar influenciada por la ausencia de servicios públicos y gratuitos de calidad.

Las representaciones sociales del cuidado en Uruguay son de tipo familistas, según muestran los antecedentes de investigación en el país (Batthyány, Genta y Perrota, 2013), lo cual se relaciona con el aumento del tiempo que las personas dedican al cuidado de los otros, tanto en sus propios hogares como en otros hogares.

Dichas representaciones del cuidado se dan en un marco donde existen políticas públicas y elementos normativos débiles para garantizar derechos de cuidado, lo cual hace que probablemente gran parte de la población uruguaya pueda siquiera imaginar alguna alternativa de calidad a los cuidados familiares y adapte sus preferencias ${ }^{18}$ en relación a estas construcciones.

En cuanto a las horas de cuidado que aportan los varones, las registradas en 2013 son mayores a las registradas en 2007 . Esto puede estar relacionado con el hecho de que los ítems de cuidado fueron medidos de manera más exhaustiva en 2013, o quizá, al hecho de que los varones efectivamente cuiden más tiempo.

A pesar de que los varones participan casi de manera incambiada entre 2007 y 2013 en el trabajo de cuidado (combinado con que se capta mejor que en 2007), las mujeres aumentaron levemente el trabajo de cuidados lo cual deja un saldo negativo en la brecha de género en la tasa de participación (casi $2 \%$ respecto a 2007), diferencia que no es estadísticamente significativa. Sin embargo, la brecha de género en el tiempo promedio dedicado al cuidado disminuye en 3 horas en el período.

Si bien a primera vista la situación respecto al trabajo doméstico y al cuidado en el período parece similar (aumenta la brecha de género en la tasa de participación y disminuye la brecha de género en el tiempo promedio dedicado al trabajo no remunerado) sabemos que las posibles tendencias a la transformación en el involucramiento en estos trabajos son bien distintas.

Jon Elster (1991) plantea el concepto de preferencias adaptativas, para indicar la declaración de una preferencia en el marco de restricciones de oportunidades. Por ejemplo, es difícil saber cómo serían las preferencias y representaciones del cuidado infantil si se contara con excelentes centros de cuidado disponibles de manera gratuita para toda la población. 
En el caso del trabajo doméstico, los varones que se involucran (14,1\% menos en 2013 que en 2007) dedican la misma cantidad de tiempo semanal en 2007 y en 2013. Por su parte, las mujeres participan en menor proporción de las tareas domésticas en 2013, que en 2007 y disminuyeron dos horas su tiempo de trabajo doméstico no remunerado. ES decir que los varones participan menos y dedican el mismo tiempo a las tareas domésticas en 2007 y 2013, mientras que las mujeres se "descargan" de trabajo doméstico en el período, probablemente comprando servicio doméstico en el mercado. En cambio en el caso del cuidado, la brecha en la tasa de participación aumenta pero de manera más leve, por que las mujeres se involucraron un poco más que los varones en el cuidado, pero la brecha de tiempo disminuye (en 2007 era de 8 horas y en 2013 es de 5) más que nada por un mayor aumento del tiempo de cuidado dedicado por los varones.

Esto muestra que mientras que resulta difícil que los varones aumenten su participación y sobre todo, su tiempo en la realización de las tareas domésticas, esta dificultad no aparece tan patente en el caso de los cuidados. En el caso del trabajo de cuidado infantil, por ejemplo, los varones mantienen incambiada su participación, pero aumentan un promedio de 5 horas semanales a la realización de las tareas de cuidado de niños y niñas. Las mujeres si aumentaron su participación en el cuidado infantil en 2007 en relación a 2013, un 1,6\% y 3 horas semanales en promedio en su realización.

En este sentido parecería que para la población masculina que se involucra en el cuidado infantil hay una valorización del tiempo dedicado a este tipo de trabajo, en la medida que van progresivamente aumentando la cantidad de horas semanales que destinan al mismo. Sin
Tabla 8. Tasa de participación (TP) , tiempo semana promedio (HS) y brechas de género y en el período del trabajo de cuidado infantil no remunerado por sexo. Total país.

\begin{tabular}{|c|c|c|c|c|c|c|}
\hline \multirow{2}{*}{} & \multicolumn{3}{|c}{ Mujeres } & \multicolumn{4}{c|}{ Varones } & \multicolumn{3}{c|}{$\begin{array}{c}\text { Brecha de } \\
\text { género }\end{array}$} \\
\cline { 2 - 7 } & TP & HS & TP & HS & TP & HS \\
\hline 2007 & 29,9 & 18 & 21,7 & 10 & 8,2 & 8 \\
\hline 2013 & 31,5 & 21 & 21,7 & 15 & 9,7 & 6 \\
\hline $\begin{array}{c}\text { Brecha } \\
2013-2007\end{array}$ & 1,6 & 3 & 0 & 5 & 1,5 & -2 \\
\hline
\end{tabular}

Fuente: elaboración propia en base a los microdatos de la Encuesta de Uso del Tiempo y Trabajo No Remunerado 2007 y 2013, módulos de la Encuesta Continua de Hogares, INE-FCSINMUJERES. Uruguay.

embargo, se encuentra el desafío de lograr un mayor involucramiento por parte de ellos, un acercamiento en las tasas de participación de varones y mujeres y un estímulo a la tendencia observada de aumentar el tiempo de cuidado por parte de los varones.

Estos indicadores podrían modificarse en la medida que la legislación sobre políticas de cuidado en Uruguay cambie. Recientemente se han creado licencias parentales que permitirían que los varones se involucren de manera más activa en los cuidados de los recién nacidos sin que esto afecte sus puestos laborales y permiten que las mujeres cuenten con la posibilidad de reintegrarse a trabajar después de cumplida la licencia maternal obligatoria.

Si bien los cambios culturales en torno al cuidado serán lentos, puesto que también son cambios de género, acciones como el aumento de la oferta 
de centros de cuidado de calidad, o el impulso a las políticas de tiempo pueden implicar avances importantes hacia la corresponsabilidad entre el Estado, el mercado, la comunidad y los hogares. Dentro de estos últimos se podría colaborar con el estímulo a un equilibrio mayor del trabajo de cuidados y doméstico aportado por los varones y las mujeres para lo cual se deben diseñar instrumentos de política orientados a la búsqueda de dicho equilibrio ${ }^{19}$.

Al igual que en el caso del trabajo doméstico, existe una tendencia a la generación de un sector de servicios de cuidado dirigido a distintas poblaciones: niños y niñas, adultos mayores y discapacitados con dependencia. Este sector está en formación y está siendo impulsado y apoyado por parte de la sociedad civil organizada (por ejemplo, la Red Pro Cuidados), aunque ha encontrado resistencias para constituirse como un grupo de presión en las negociaciones sindicales y las centrales de trabajadores (Aguirre, 2014).

La falta de organización para constituir un "Sector" de cuidados como rama de actividad económica y los salarios que el Estado está pensando para los cuidadores contratados por el incipiente Sistema Nacional de Cuidados, reproducen, a través de la desvalorización económica, una desvalorización del cuidado como un trabajo 20 .

\footnotetext{
9 Un estudio realizado recientemente sobre el impacto de las licencias parentales (Batthyány, Genta y Perrotta, 2015) muestra que la medida no colabora a modificar la actual división sexual del trabajo en los cuidados.

20 Como evidencia la Tabla 9 en anexos, el trabajo remunerado de cuidados está altamente feminizado ( $94,4 \%$ son mujeres), de las cuales casi el $50 \%$ no aporta a la caja de jubilaciones, ganan menos de la mitad del salario promedio de la población ocupada en 2014 y la incidencia de la pobreza es un 56\% más alta (3,3 puntos porcentuales de diferencia) que la registrada en la población ocupada total.
}

\subsection{Variaciones en la dedicación a los diferentes componentes del trabajo no remunerado realizado para otros hogares y voluntario en Uruguay, 2007-2013.}

Dentro del trabajo no remunerado no se encuentran solo las actividades domésticas y de cuidados realizadas por las personas para sus propios hogares, sino que también se estima el trabajo aportado para otros hogares. En general, la participación de varones y mujeres en el trabajo doméstico y de cuidados en los hogares de otros se redujo en el período 2007-2013. Dicha reducción es levemente mayor entre las mujeres que en los varones. En cuanto al tiempo, entre 2007 y 2013, tanto varones como mujeres aumentaron un promedio de 9 horas semanales de trabajo en relación al promedio que se había captado con la Encuesta de Uso del Tiempo de 2007 y no hubo modificaciones en la brecha de género de tiempo dedicado a este tipo de trabajo. Las mujeres siguen aportando 4 horas en promedio más que los varones al trabajo no remunerado para otros hogares.

El trabajo inter-hogares, muestra que el trabajo no remunerado no es un trabajo atomizado en las unidades que representan los hogares, sino que es un verdadero trabajo en redes familiares (a veces, con la participación de amigos o vecinos). Las mismas son importantísimos agentes productores de bienestar social, que aportan soluciones de trabajo necesarias para sostener la vida cotidiana.

Respecto de la tasa de participación en el trabajo no remunerado voluntario, los varones han aumentado un poco más que las mujeres su declaración en el período, aunque el tiempo dedicado al voluntariado ha aumentado 7 horas tanto para varones como para mujeres. 
Tabla 9. Tasa de participación (TP) , tiempo semanal promedio (HS) y brechas de género y en el período del trabajo no remunerado brindado a otros hogares por sexo en 2007 y 2013. Total país.

\begin{tabular}{|c|c|c|c|c|c|c|}
\hline \multirow{2}{*}{} & \multicolumn{2}{|c|}{ Mujeres } & \multicolumn{2}{c|}{ Varones } & \multicolumn{2}{c|}{$\begin{array}{c}\text { Diferencias } \\
\text { MN }\end{array}$} \\
\cline { 2 - 7 } & TP & HS & TP & HS & TP & HS \\
\hline 2007 & 10,4 & 14 & 4,6 & 10 & 5,8 & 4 \\
\hline 2013 & 8,7 & 23 & 4,4 & 19 & 4,3 & 4 \\
\hline $\begin{array}{c}\text { Brecha } \\
2013-2007\end{array}$ & $-1,7$ & 9 & $-0,2$ & 9 & $-1,5$ & 0 \\
\hline
\end{tabular}

Fuente: elaboración propia en base a los microdatos de la Encuesta de Uso del Tiempo y Trabajo No Remunerado 2007 y 2013, módulos de la Encuesta Continua de Hogares, INE-FCSINMUJERES. Uruguay.

Tabla 10. Tasa de participación (TP) tiempo semanal promedio (HS) y brechas de género y en el período del trabajo no remunerado brindado a otros

hogares por sexo en 2007 y 2013. Total país.

\begin{tabular}{|c|c|c|c|c|c|c|}
\hline \multirow{2}{*}{} & \multicolumn{2}{|c|}{ Mujeres } & \multicolumn{2}{c|}{ Varones } & \multicolumn{2}{|c|}{$\begin{array}{c}\text { Diferencias } \\
\text { M/N }\end{array}$} \\
\cline { 2 - 7 } & TP & HS & TP & HS & TP & HS \\
\hline 2007 & 3,1 & 10 & 1,9 & 9 & 1,2 & 1 \\
\hline 2013 & 3,9 & 17 & 3,4 & 16 & 0,5 & 1 \\
\hline $\begin{array}{c}\text { Brecha } \\
2013-2007\end{array}$ & 0,8 & 7 & 1,5 & 7 & $-0,7$ & 0 \\
\hline
\end{tabular}

Fuente: elaboración propia en base a los microdatos de la Encuesta de Uso del Tiempo y Trabajo No Remunerado 2007 y 2013, módulos de la Encuesta Continua de Hogares, INE-FCSINMUJERES. Uruguay.

\section{Reflexiones finales.}

En el artículo se ha mostrado brevemente algunas de las persistencias y cambios en la división sexual del trabajo en Uruguay entre 2007 y 2013, destacándose una mayor diferenciación en la participación en el trabajo doméstico, de cuidados y voluntario por parte de varones y mujeres, pero con un acortamiento de las brechas del tiempo promedio dedicado por aquellos que efectivamente participan del trabajo no remunerado.

Se ha partido de la base de que el tiempo y el trabajo son dos grandes indicadores que ayudan a conocer como es la actual división sexual del trabajo en Uruguay y en la región. Permiten conocer en qué ámbitos se desempeñan los varones y las mujeres, si dicho desempeño es equitativo o no y qué costos y beneficios están asociados a los diferentes escenarios de trabajo.

Mediante la realización del trabajo se ha constatado limitaciones en las fuentes de datos para poder comparar los datos recogidos en distintos momentos del tiempo, lo cual limita la capacidad de análisis y de desagregación de los datos.

Las mismas permitirían realizar un análisis comparado en el tiempo que permitiera integrar otros aspectos como las dinámicas y estructuras familiares, centrales para el abordaje de la división sexual del trabajo, pero cuyas categorías demandan datos con mayores muestras y comparables por variables como tipo de hogar, con lo que no contamos en este momento.

Es por esto que es importante reconocer las limitaciones de las Encuestas de Uso del Tiempo utilizadas, y de resaltar lo importante que es 
dicho instrumento para conocer aspectos medulares de las relaciones de género en las sociedades actuales.

Se considera que es necesario velar por la comparabilidad de las EUTs y caminar hacia estudios panel que permitan controlar por cohorte, período y edad.

En términos generales, se ha visualizado que en Uruguay hay una persistente división sexual del trabajo en la que las mujeres se incorporan progresivamente al mercado de empleo pero los varones no solo no se incorporan sino que se retiran de la participación en el trabajo no remunerado.

Dentro de los costos que pagan las mujeres por realizar trabajo de cuidados y doméstico no pago y con escasa valoración social se destacan: la dificultad de acceso a ingresos propios presentes (ausencia o disminución de la participación en el mercado laboral) y futuros (bajas jubilaciones y pensiones en la vejez debido a sus trayectorias laborales pasadas); dificultad de acceso a derechos sociales que son canalizados a través del trabajo remunerado; desvinculación del sistema educativo; malas condiciones de salud física y psíquica (cuando se realizan tareas de cuidado, particularmente a enfermos), carencia de tiempo libre y de descanso, entre otros.

Por otra parte, se ha mostrado que si bien la participación en el TNR aumenta las brechas de género en el período, en el tiempo promedio se da un fenómeno contrario y el mismo es bien distinto cuando se trata del trabajo de cuidados que cuando se trata del trabajo doméstico. Los varones se resisten con más fuerza a realizar el segundo tipo de trabajo, despojado del afecto y los elementos vinculares y emocionales del primero. Su participación en los cuidados (para la que se registra una tendencia en el aumento de la dedicación de tiempo) pone de manifiesto que el cuidado puede ser una puerta de entrada a la participación masculina en el trabajo no remunerado.

Parte de la disminución de las brechas de tiempo está vinculada con un aumento de las horas dedicadas por los varones que se involucran activamente en el cuidado. En este sentido, se considera que es posible desde las políticas públicas generar estímulos para alentar el compromiso de los varones con el trabajo no remunerado de cuidados, pero también doméstico y voluntario.

A su vez, el Estado puede contribuir a la profesionalización del trabajo de cuidados, que también tiende a su mercantilización, estimulando buenas condiciones laborales con el fin de otorgar reconocimiento y valorización al trabajo de cuidados.

En síntesis Uruguay está posicionado como un país referente en los avances formales en la equidad de género y en la politización del tema, sin embargo persiste en el país una división sexual del trabajo clásica que implica la puesta en marcha de propuestas creativas y variadas para trabajar en torno a pautas culturales patriarcales arraigadas de manera virtuosa en la cultura, las instituciones, el intercambio de expectativas de género y las subjetividades. De esta forma, se avanzaría en la equidad entre varones y mujeres en el trabajo remunerado y no remunerado lo que posicionaría a Uruguay como un país más equitativo en términos reales. 


\section{Bibliografía}

Adelantado, J. et al. 1998. "Las relaciones entre estructura y políticas sociales: una propuesta teórica". Revista Mexicana de Sociología 3: 123-156.

Aguirre Miguélez, A. 2016. "Negociaciones de pareja: los trabajos domésticos, la crianza y la construcción de la maternidad y la paternidad". Papeles del CEIC 152: 1-27.

Aguirre, R. 2003. Género, ciudadanía social y trabajo. Montevideo: Doble Clic Editoras.

2009. Las bases invisibles del bienestar social. El trabajo no remunerado en Uruguay. Montevideo: Doble Clic Editoras.

\section{Personas ocupadas en el sector cuidado.} Montevideo: MIDES, Serie Sistema Nacional de Cuidados.

Aguirre, R y Batthyány, K. 2005. Uso del tiempo y trabajo no remunerado. La encuesta Montevideo y Área Metropolitana 2003. Montevideo: UNIFEM, UDELAR.

Aguirre, R. et al. 2014. "Los cuidados en la agenda de investigación y en las políticas públicas en Uruguay". Iconos. Revista de Ciencias Sociales 50: 43-60.

Araya, M.J. 2003. Un acercamiento a las Encuestas sobre el Uso del Tiempo con orientación de género. Santiago de Chile: CEPAL, Serie Mujer y Desarrollo 50.

Arriagada, I. 1997. Políticas Sociales, Familia y Trabajo en la América Latina de fin de Siglo. Santiago de Chile: CEPAL, Serie Políticas Sociales 21.

Batthyány, K. 2015. Los tiempos del bienestar social. Género, trabajo no remunerado y cuidados en Uruguay. Montevideo: Doble Clic Editoras.

Batthyány, K. et al. 2013. La población uruguaya y el cuidado. Análisis de representaciones sociales y propuestas para un Sistema de Cuidados en Uruguay. Montevideo: MIDES.

Batthyány, K. etal.2015. Avanzando hacia la corresponsabilidad en los cuidados Análisis de las licencias parentales en el Uruguay. Santiago de Chile: CEPAL, Serie Asuntos de Género 128.

Beck, U. y Beck-Gernsheim, E. 2012. Amora distancia. Nuevas formas de vida en la era global. Argentina: Paidós contextos.

Bock, G. y Duden, B. 1985. "Trabajo por amor, amor como trabajo". Desarrollo, Revista de la Sociedad Internacional para el desarrollo 2: 4-14.

Carrasco, C. Et al. 2011. "Introducción. El trabajo de cuidados: antecedentes históricos y debates actuales". El trabajo de cuidados. Historia, teorías y política. Carrasco, C. Et al. (Eds.). Madrid: Los libros de la catarata.13-96

Delfino, A. 2009. "La metodología de uso del tiempo: sus características, limitaciones y potencialidades". Espacio Abierto 2: 199-218.

Daly, M. y Jane, L. 2000. "The concept of social care and the analysis of contemporary welfare states". British Journal of Sociology. 51: 281-298.

Daly, M. y Jane, L. 2011. "El concepto de "Social Care" y el análisis de los estados de bienestar contemporáneos". El trabajo de cuidados. Historia, teorías y política. Carrasco, C. Et al. (Eds.). Madrid: Los libros de la catarata.

Durán, M.A. 1988. De puertas adentro. Madrid: Ministerio de Cultura, Instituto de la Mujer.

2006. La Cuenta Satélite del Trabajo No Remunerado en

la Comunidad de Madrid, Consejería de Empleo y Mujer, Dirección General de la Mujer, Madrid. Disponible en: http://www.madrid. org/cs/Satellite?c=CM_Publicaciones_FA\&cid $=1142310100$ 275\&idTema $=1109265587163 \&$ language $=$ es\&pagename=Comuni dadMadrid\% 2FEstructura\&segmento=1\&sm $=1$

Elster, J. 1991. Tuercas y tornillos. Una introducción a los conceptos básicos de las ciencias sociales. Barcelona: Gedisa.

Elías, N. 1989. Sobre el tiempo. México: Fondo de Cultura Económica.

Esping Andersen, G. 1990. The Three Worlds Of Welfare Capitalism. Cambridge: Polity Press. 9-54.

2000. Fundamentos sociales de las economías postindustriales. Madrid: Ariel Sociología.

Faur, E. 2014. El cuidado infantil en el siglo xxi. Mujeres malabaristas en una sociedad desigual. Buenos Aires: Siglo XXI Editores.

Giddens, A. 2000. Sociología. Madrid: Alianza Editorial.

Gómez Luna, M.E. 2003. "Macroeconomía y trabajo no remunerado". Economía y género: macroeconomía, política fiscal y liberalización; análisis de su impacto sobre las mujeres. De Villota, P. Barcelona: Icaria. 159-208.

2010. Directrices y referentes conceptuales para armonizar las encuestas sobre uso del tiempo en América Latina y el Caribe. Documento para discusión. Conferencia estadística de las Américas de la comisión económica para América Latina y el Caribe. México D. F Julio. Inmujeres-Unifem-Cepal-Inegi.

Himmelweit, S. 1995. "The discovery of "unpaid work": the social consequences of the expansion of "work". Feminist Economics 1:1-19.

INE. 2015a. Anuario Estadístico 2015. Montevideo: INE. Disponible en: http://www.ine.gub.uy/documents/10181/351713/ Anuario+Estad\%C3\%ADstico+2015/9b97bb4e-f863-4ffc-bd62b5356b3aa732

INE. 2015b. Mujeres y hombres en España. Madrid: INE. Disponible en: http://www.ine.es/ss/Satellite?L=es_ES\&c=INEPu blicacion_C\&cid=1259926137287\&p=1254735110672\&pagenam e=ProductosYServicios\%2FPYSLayout\&param1=PYSDetalleGra tuitas

INMUJERES. 2015. Estadísticas de Género 2014. Avances y desafíos para la equidad de género. Montevideo: MIDES. Disponible en: http://www.inmujeres.gub.uy/innovaportal/ file/57783/1/estadisticas-de-genero-2014.pdf

Martínez Buján, R. 2010. "La reorganización de los cuidados familiares en un contexto de migración internacional" Cuadernos 
de Relaciones Laborales. 29: 93-123.

Martinez, J. y Voorend, K. 2013. "Desigualdades de género en los regímenes de bienestar latinoamericanos: mercado, política social y organización familiar de los cuidados". Las fronteras del cuidado. Agenda, derechos e infraestructura. Pautassi, L u Siebecchi, C. (Eds) Buenos Aires: Editorial Biblos. 59-98.

Legarreta, I. 2011. "El tiempo donado en el ámbito doméstico. Reflexiones para el análisis del trabajo doméstico y los cuidados". El trabajo y la ética del cuidado. Arango Gaviria, L.G y Molinier, P. (Comp) Medellín: La carreta editores.

Lewis, J. 1997. "Gender and Welfare Regimes: Further Thoughts". Social Politics. Oxford University Press.

OIT. 2013. Estadísticas del trabajo y de la fuerza de trabajo Informe para el debate en la Reunión de expertos en estadísticas del trabajo para el avance de las estadísticas sobre el empleo y el desempleo. Ginebra: Departamento de Estadística.

OPS, CEPAL, CSIC. 2008. La economía invisible y las desigualdades de género. La importancia de medir y valorar el trabajo no remunerado. Washington: OPS.

Pateman, C. 1990. "Feminismo y democracia". Debate Feminista 1 (1).

Salvador, S. 2009. "La valorización económica del trabajo no remunerado". Las bases invisibles del bienestar social. Aguirre, R.
(Coord). Montevideo: Doble Clic Editoras. 155-203.

2015. "La valorización económica del trabajo no remunerado". Los tiempos del bienestar social. Batthyány, K. (Coord) Montevideo, Doble Clic Editoras. 212-242.

Sainsbury, D. 2000. "Les droits sociaux des femmes et des hommes. Les dimensions de genre dans les états providence" Genre et politique. Debats et perspectives. France: Folio Essais Gallimard.

Saraceno, C. 1994. "A dependencia construida e a independencia negada: estruturas de genero da cidadania" $O$ dilema da cidadania, Bonacchi, G. y Groppi, A. (Eds).San Pablo: Unesp.

SEDESOL. 2010. Diagnóstico de la problemática de las madres con hijos pequeños para acceder o permanecer en el mercado laboral. México: SEDESOL.

SIG-Inmujeres. 2013. Estadísticas de Género 2013. Evolución de los indicadores de género en el período 2009-2013. Montevideo: MIDES-Inmujeres- UNFPA.

Tobío, C. 2005 Madres que trabajan. Dilemas y estrategias. Madrid: Ediciones Cátedra.

Torns, T. 2008. "El trabajo y el cuidado: cuestiones teóricometodológicas desde la perspectiva de género". EMPIRIA. Revista de Metodología de Ciencias Sociales 15: 53-73. 